\title{
Exact modelling of generalised defect modes in photonic crystal structures
}

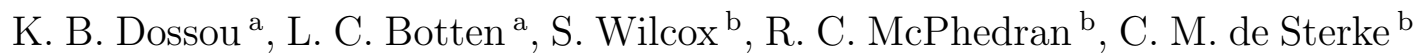 \\ N. A. Nicorovici ${ }^{a, b}$ A. A. Asatryan ${ }^{a}$ \\ ${ }^{a}$ Centre for Ultrahigh-bandwidth Devices for Optical Systems (CUDOS) and Department of Mathematical Sciences, \\ University of Technology, Sydney, PO Box 123, Broadway, NSW 2007, Australia \\ ${ }^{\mathrm{b}}$ CUDOS and School of Physics, University of Sydney, NSW 2006, Australia
}

\begin{abstract}
An exact theory for modelling modes of generalised defects in 2D photonic crystals (PCs) with a genuinely infinite cladding is presented. The approach builds on our fictitious source superposition method for simple defects and permits an elegant extension allowing the modelling of arbitrary defects. Numerical results that demonstrate the accuracy and efficiency of the extended method are presented. We also use the method to study the evolution of the mode generated by varying the refractive index of a single defect cylinder and find significant differences between the behaviour of defects in rod-type and hole-type PCs.

Keywords: waveguides, photonic crystals, defect modes, resonant cavities.
\end{abstract}

\section{Introduction}

While band gaps provide the mechanism by which light is confined within a photonic crystal (PC), it is the introduction of defects (e.g., waveguides, cavities etc.) into structures that are otherwise periodic that allows interesting and useful devices to be constructed [1]. The introduction of one or more defects can shift propagating modes in photonic bands to localised modes whose frequencies lie within a band gap of the surrounding bulk PC. Since a defect breaks the periodicity of the PC, Bloch's theorem no longer holds and so the usual methods for computing band structures (which reduce the computational domain to a single unit cell) are not applicable. Typically, the numerical modelling of defect modes in PCs is based on either a supercell approach, whereby a finite structure is replicated periodically, or by the truncation of an unbounded problem domain to a finite domain using absorbing boundary conditions. While such techniques work well in the majority of

Preprint submitted to Elsevier cases, the computational requirements can be prohibitive when the computational domain required to model the mode accurately needs to be large - such as in the case of a mode near cut-off when it can become arbitrarily extended.

In order to deal with this class of problems, we developed an exact theory [2], known as the fictitious source superposition (FSS) method, for computing defect modes in an infinite $2 \mathrm{D}$ lattice, applying it to the study of the long-wavelength behaviour of PC fibres (PCFs) [3], and demonstrating unambiguously that the fundamental mode was never cut off. While our original implementation [2] was a useful tool, it is nevertheless limited to handling only simple (single cylinder) defects. Accordingly, here, we outline a generalisation of the FSS method that can accommodate complex defects comprising the removal or modification of a multiplicity of cylinders (in either a single or multiple rows) of an infinite PC. In Section 2, we outline the principles of the FSS method and of the generalisation of the theory for a complex defect. Section 3 presents some numerical sim- 


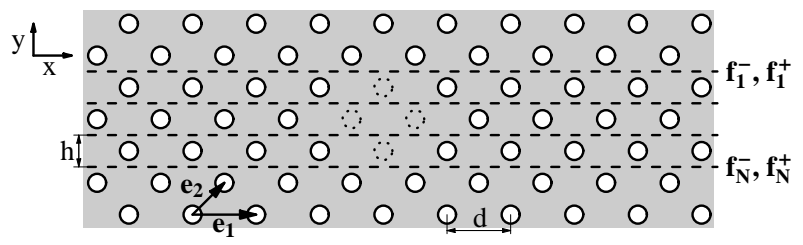

Fig. 1. Diagram of a complex defect formed by the removal of a single cylinder in each of two layers and a pair of cylinders in a third layer.

ulations in which we demonstrate the reliability and accuracy of the method, as well as investigating the evolution of defect modes by varying the refractive index of a single cylinder.

\section{Theory}

We consider 2D PCs with defects formed by removing (or modifying) a finite number of cylinders in adjacent rows of the regular lattice (see Fig. 1). While the theory has been implemented for the general (conical incidence) case in which the axial field dependence is $\exp (i \beta z)$, here, for the sake of simplicity, we outline only the in-plane treatment, reducing the fully vectorial problem to one which may be characterised by a single scalar field component$V=E_{z}$ for TM polarisation, and $V=H_{z}$ for TE polarisation. The field $V$ satisfies the scalar Helmholtz equation $\left(\nabla^{2}+k_{j}^{2}\right) V=0$, where $k_{j}$ denotes the wave number in medium $j$. Thus, in the vicinity of each cylinder (centred on $\boldsymbol{r}=\boldsymbol{r}_{j}$ ), and also within the interior of this cylinder, we can respectively expand the field in multipole expansions [4]

$$
\begin{aligned}
& V_{e}=\sum_{n=-\infty}^{\infty}\left[a_{n}^{j} J_{n}\left(k_{e} \rho\right)+b_{n}^{j} H_{n}^{(1)}\left(k_{e} \rho\right)\right] e^{i n \theta}, \\
& V_{i}=\sum_{n=-\infty}^{\infty}\left[c_{n}^{j} J_{n}\left(k_{i} \rho\right)+q_{n}^{j} H_{n}^{(1)}\left(k_{i} \rho\right)\right] e^{i n \theta}
\end{aligned}
$$

using local cylindrical coordinates $(\rho, \theta)=\boldsymbol{r}-\boldsymbol{r}_{j}$, with $k_{e}=k n_{e}$ and $k_{i}=k n_{i}$ respectively denoting the exterior and interior wave numbers, and with the free space wave number given by $k=2 \pi / \lambda$. We formulate the problems using matrix notation; vectors of coefficients and matrices will be represented by boldface symbols, for instance $\boldsymbol{b}^{j}=\left[b_{n}^{j}\right]$. The $\boldsymbol{b}^{j}$ coefficients (1) characterise the outward radiation from cylinder $j$, while the $\boldsymbol{a}^{j}$ coefficients define the regular part of the field which comprises radiation incident due to either exterior sources, or radiation from other cylinders in the cluster. Correspondingly, in Eq. (2) the regular part of the field in the interior of the scatterers is characterised by coefficients $\boldsymbol{c}^{j}$, while the coefficients $\boldsymbol{q}^{j}$ represent a fictitious source that we may use to tailor the external field in order to mimic one caused by a physical defect (i.e., the absence or modification of any cylinder). This is the first of the three main ideas underlying the FSS method. To see how this works, we initially consider a single cylinder and note that the field continuity conditions at the cylinder boundary impose the following relations between the field coefficients [5]

$\boldsymbol{b}^{j}=\hat{\boldsymbol{R}} \boldsymbol{a}^{j}+\hat{\boldsymbol{T}} \boldsymbol{q}^{j}, \quad \boldsymbol{c}^{j}=\hat{\boldsymbol{T}}^{\prime} \boldsymbol{a}^{j}+\hat{\boldsymbol{R}}^{\prime} \boldsymbol{q}^{j}$.

The explicit forms of the scattering matrices $\hat{\boldsymbol{R}}, \hat{\boldsymbol{T}}$, $\hat{\boldsymbol{T}}^{\prime}$ and $\hat{\boldsymbol{R}}^{\prime}$ can be found in Ref. [5]. Now, to remove, in effect, cylinder $j$ in the presence of the incident field $\boldsymbol{a}^{j}$, we require the radiated field $\boldsymbol{b}^{j}=0$. This we may achieve with a fictitious source $\boldsymbol{q}^{j}=$ $-\hat{\boldsymbol{T}}^{-1} \hat{\boldsymbol{R}} \boldsymbol{a}^{j}$.

The removal of a single cylinder is straightforward if the incident field is known. In general, however, for an infinite array, it is not possible to compute this from first principles and so we adopt an alternative approach, formulating the defect mode as a superposition of solutions of quasiperiodic field problems, allowing us to solve the problem on the domain of a single unit cell. We thus consider an array of cylinders containing embedded sources which are quasiperiodically phased, i.e., we set the source in cylinder $j$ centred at $\boldsymbol{r}=\boldsymbol{r}_{j}$ to be $\boldsymbol{q}^{j}=\boldsymbol{q} \exp \left(i \boldsymbol{k}_{0} \cdot \boldsymbol{r}_{j}\right)$ where the omission of the subscript $j$ corresponds to $j=0$. The defect mode is then formed from a superposition of these quasiperiodic problems by integrating with respect to the Bloch vector $\boldsymbol{k}_{0}$ over the first Brillouin zone (BZ) of the reciprocal lattice. The superposed solution then satisfies the wave equation and the boundary conditions, and is associated with a fictitious source distribution which, for cylinder $j$, is

$\frac{\boldsymbol{q}}{A_{\mathrm{BZ}}} \int_{\mathrm{BZ}} \exp \left(i \boldsymbol{k}_{0} \cdot \boldsymbol{r}_{j}\right) d \boldsymbol{k}_{0}=\left\{\begin{array}{l}\boldsymbol{q} \text { for } \boldsymbol{r}_{j}=0, \\ \mathbf{0} \text { for } \boldsymbol{r}_{j} \neq 0,\end{array}\right.$

where $A_{\mathrm{BZ}}$ denotes the area of the BZ. In this step, the BZ integration eliminates the fictitious source in all but the primary cylinder $(j=0)$, with the remaining source at $\boldsymbol{r}=\boldsymbol{r}_{0}=\mathbf{0}$ available to modify the response field and so formulate the defect mode.

The first extension we outline to the formulation of Wilcox et al [2] enables it to handle multiple defects. To do this, we write 
$\boldsymbol{q}=\sum_{m} \boldsymbol{q}_{m} \exp \left(-i \boldsymbol{k}_{0} \cdot \boldsymbol{r}_{s_{m}}\right)$

with $s_{m} \in \mathcal{S}$, where $\mathcal{S}$ denotes the set of cylinders to be modified or removed. The source at cylinder $j$ is again $\boldsymbol{q}^{j}=\boldsymbol{q} \exp \left(i \boldsymbol{k}_{0} \cdot \boldsymbol{r}_{j}\right)$ and the integration over the BZ in Eq. (4) now preserves all sources in the chosen set $\mathcal{S}$, since $\left\langle\boldsymbol{q}^{j}\right\rangle=0$ at all $j$ except those $j \in$ $\mathcal{S}$ for which $\left\langle\boldsymbol{q}^{j}\right\rangle=\left\langle\boldsymbol{q}^{s_{m}}\right\rangle=\boldsymbol{q}_{m}$. These remaining sources are thus available to tailor the field and, in doing so, to introduce defects.

In the superposition step (4), the need to undertake a $2 \mathrm{D}$ integration over the $\mathrm{BZ}$ imposes a heavy computational burden. We may reduce this to a $1 \mathrm{D}$ integration by reformulating the problem using a diffraction grating model, thus improving the computational efficiency greatly. To achieve this, we model the structure as a diffraction grating (or, in general, multiple gratings) having embedded quasiperiodically phased array(s) of sources, sandwiched between two semi-infinite PCs which act as mirrors, confining the light by a band gap. Figure 1 shows an example of a defect that spans three grating layers placed between two semi-infinite PCs. Since details of the basic grating model for the single defect are given in our earlier paper [2], we describe here only the additional elements needed to treat defects in several different rows of cylinders in a PC.

For a defect spanning $n$ grating layers, vectors of coefficients are partitioned between the layers; for instance, the symbols $\boldsymbol{b}_{l}$ and $\boldsymbol{q}_{l}$ represent respectively the vectors $\boldsymbol{b}^{j}$ and $\boldsymbol{q}^{j}$ associated to a layer $l$. Since we consider a quasiperiodic array of sources in each row to be modified, each gratings act as a source of outward (i.e., upward and downward) propagating plane waves. These outgoing plane waves are scattered (reflected and transmitted) by other layers which, if they contain sources, also generate outgoing waves. By carefully following the scattering processes in a recursive manner[7], we may write that for layer $l$ containing embedded sources $\left(\boldsymbol{q}_{l}\right)$, the outgoing multipole coefficients $\left(\boldsymbol{b}_{l}\right)$ are

$\boldsymbol{b}_{l}=\boldsymbol{Y}_{-} \boldsymbol{f}_{l}^{-}+\boldsymbol{Y}_{+} \boldsymbol{f}_{l+1}^{+}+\boldsymbol{Q}_{l} \boldsymbol{q}_{l}$,

indicating that the outgoing multipole field is generated by incoming plane waves immediately above $\left(\boldsymbol{f}_{l}^{-}\right)$and below $\left(\boldsymbol{f}_{l+1}^{+}\right)$the layer, and by embedded sources. In turn, the outgoing plane wave fields $\left(\boldsymbol{f}_{l}^{+}\right.$ and $\boldsymbol{f}_{l+1}^{-}$) from layer $l$ may be expressed in terms of the incident plane wave fields $\left(\boldsymbol{f}_{l}^{-}\right.$and $\left.\boldsymbol{f}_{l+1}^{+}\right)$and the outgoing field generated by cylinders in this particular layer that depends on $\boldsymbol{b}_{l}$. Finally, we close the system by applying the mirror conditions for the semi-infinite PCs that sandwich the source layers, which we model using a (plane wave) reflection matrix $\boldsymbol{R}_{\infty}$ that follows from a Bloch mode analysis of the bulk PC [6]. In this way, outgoing radiation from embedded source layers is reflected back into the system, thereby relating the outgoing multipole coefficients $\left\{\boldsymbol{b}_{l}\right\}$ in each layer with the embedded fictitious sources $\left\{\boldsymbol{q}_{m}\right\}$ in all layers. This may be expressed formally in the linear system

$\boldsymbol{b}=\boldsymbol{Z q}$,

in which $\boldsymbol{b}=\left[\boldsymbol{b}_{l}\right], \boldsymbol{q}=\left[\boldsymbol{q}_{m}\right]$ and $\boldsymbol{Z}=\left[\boldsymbol{Z}_{l m}\right]$ are partitioned vectors and matrices.

It is Eq. (7) that allows us to compute the defect modes. In it, $\boldsymbol{Z}=\boldsymbol{Z}\left(k, \alpha_{0}\right)$, where $k$ is the wave number and $\alpha_{0}=\boldsymbol{k}_{0 x}$. The simplest calculation is for a waveguide, formed by removing an entire row of cylinders. We do this by setting $\boldsymbol{b}=\mathbf{0}$, so that no outgoing wave radiates from any of the cylinders (by quasiperiodicity), and proceed to compute the dispersion curve of the waveguide by solving $\operatorname{det} \boldsymbol{Z}\left(k, \alpha_{0}\right)=0$ for $\alpha_{0}$.

As alluded to earlier, the calculation of a cavity defect mode requires the superposition of the quasiperiodic solutions. Computing this superposition by integrating over the BZ, we deduce that $\langle\boldsymbol{b}\rangle=\langle\boldsymbol{Z}(k)\rangle \boldsymbol{q}$ where

$\langle\boldsymbol{Z}(k)\rangle=\frac{d}{2 \pi} \int_{-\pi / d}^{\pi / d} \boldsymbol{Z}\left(k, \alpha_{0}\right) d \alpha_{0}$.

The defect mode is then formed by setting $\langle\boldsymbol{b}\rangle=0$ and searching for roots of $\operatorname{det}\langle\boldsymbol{Z}(k)\rangle$ to determine the eigenfrequency. In turn, the mode may be reconstructed from $\boldsymbol{q}$ which lies in the null space of $\langle\boldsymbol{Z}(k)\rangle$ at this frequency.

\section{Numerical results}

For our first example, we apply the FSS method to the calculation of cavity modes. We consider a hole-type $\mathrm{PC}$ in TE polarised light, consisting of cylinders of normalised radius $a / d=0.2$, and refractive index $n_{c}=1$ arranged in a hexagonal array, in a background of refractive index $n_{b}=3$. We consider a cavity formed by the removal of four cylinders lying on the vertices of a unit cell. The four defect cylinders can be arranged either as a set of two cylinders in each of two adjacent rows or a set 
(a)

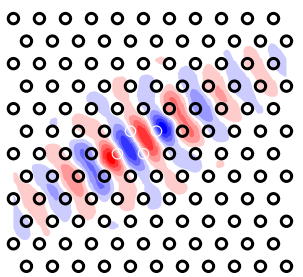

(b)

00000000000 00000000000

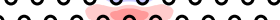

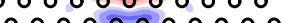
- 0000000000

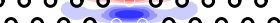

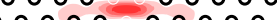

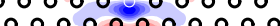
0 0 0 0 0 0 0 0 0 0 0000000000 0 0 0 0 0 0 0 0 0 00000000000

Fig. 2. Plot of the defect mode associated to the resonance frequency $d / \lambda_{1}=0.228885$ for the cavity formed by the removal of four cylinders. The four defect cylinders can be represented either in (a) two layers or (b) three layers.

of respectively one, two and one cylinders in three consecutive rows (see Fig. 2). The periodic structure has a band gap for the normalised frequency $d / \lambda \in$ $[0.225553,0.237806]$. As a test of robustness, we apply the FSS method using the two different grating layer configurations; the two approaches give exactly the same resonance frequencies (up to rounding error). There are three resonance frequencies within the band gap: $d / \lambda_{1}=0.228885, d / \lambda_{2}=0.230456$ and $d / \lambda_{3}=0.231709$. Figure 2 shows the field of the mode associated to the frequency $d / \lambda_{1}$.

For our next example, we study the evolution of the defect mode with varying refractive index of one defect cylinder. We consider both rod-type (in TM polarisation) and hole-type (in TE polarisation) PCs in a hexagonal lattice. We assume that a simple defect cylinder is introduced by changing the refractive index of a single cylinder to a value $n_{d}$. We compute the corresponding defect mode using the FSS method. For the rod-type PCs, the refractive index of the unperturbed cylinders is $n_{c}=3$, the background refractive is $n_{b}=1$ and the normalised radius is $a / d=0.2$. The structure has a band gap for normalised frequency $d / \lambda \in[0.31312,0.480299]$. In Fig. 3 (a), we plot the resonance frequency as a function of $n_{d}$. As discussed by Joannopoulos et al [1], when $n_{d}>n_{c}$ the defect mode has odd symmetry with respect to the axis of the defect cylinder and originates from the upper edge of the band gap. In the opposite case, $n_{d}<n_{c}$, the field has even symmetry and the defect mode emerges from the lower band gap edge. Note that the two modes discussed are actually doubly-degenerate. However, the FSS method exactly incorporates the lattice symmetry and so their degenerate frequencies coincide to very high accuracy.

Our code is written in Mathematica [8] which calls a Fortran routine through MathLink to compute the

scattering matrices, from which the Bloch modes of the bulk PC structure are obtained. Convergence of the resonance frequency to seven significant digits can be achieved with the multipole expansions (1) and (2) truncated to Bessel function orders in the range -3 to 3 , the plane wave orders in Eq. (6) truncated to the range -3 to 3 , and the Brillouin zone integration Eq. (8) approximated by a 60 -point Gaussian quadrature. With these parameter values, it takes about 4 seconds to compute $\operatorname{det}\langle\boldsymbol{Z}(k)\rangle$ on a Pentium $4(2.8 \mathrm{GHz})$ computer and with a good initial guess a root of the equation $\operatorname{det}\langle\boldsymbol{Z}(k)\rangle=0$ can be obtained in about 50 seconds.

For comparison, we repeat the calculation using a finite element method [9] (FEM); Fig. 3 (a) shows a good agreement between the results from both methods. However with the FEM, the computer memory requirement and the computation time increase quickly with the size of the two dimensional supercell and we have not be able to achieve very accurate results as with the FSS method. In particular, there is a slight splitting between the FEM results for the two degenerate modes. For the results presented in Fig. 3 (a), we use a rectangular computational domain of size $3 \sqrt{3} \times 5$ and impose periodic boundary conditions (supercell) at the boundaries of the domain. The finite element mesh has 9308 triangles and 18869 nodes (quadratic elements) and it takes about 20 seconds to solve the eigenvalue problem.

Note that the data of Fig. 3 (a) shows that the defect state emerges from the edge of the band, with the wavelength difference between the defect mode and the band edge tending to zero as the refractive index difference tends down to zero. Economou [10] considers defect states for the Schrödinger equation in two dimensions, and gives an exponential form going as the reciprocal of the perturbing potential for the energy difference between the band edge and the defect state. An initial investigation using the data of Fig. 3 (a) confirms this simple model for TM polarisation.

For hole-type structures, the bulk PC is the same as that of Fig. 2. As in the rod-type case, the symmetry of the mode field is even for $n_{c}<n_{d}$ and odd for $n_{c}>n_{d}$. Once again, we see from the FSS method that the defect modes emerge from the gap edge. While the plateau in $n_{d}$ around the matrix index is somewhat wider for TE polarisation than for TM polarisation, the data of Fig. 3 (b) is in good agreement with the asymptotics of Economou's defect model[10]. With TE polarised light, the FEM 


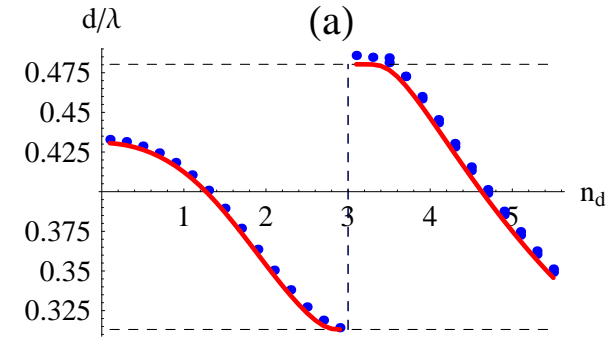

$\mathrm{d} / \lambda$

(b)

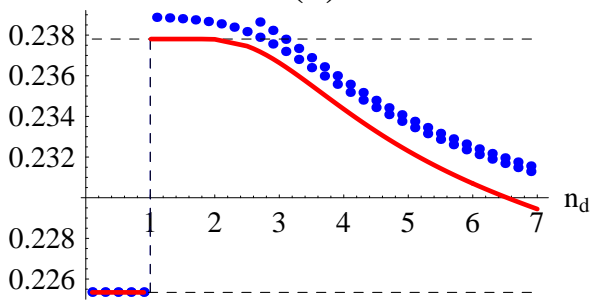

Fig. 3. Evolution of the defect mode when the refractive index of one cylinder is changed to $n_{d}$. The continuous line and the dots represent respectively the FSS and FEM results; the vertical dashed lines indicate the refractive index $n_{c}$ of the unperturbed cylinders while the horizontal dashed lines show the edges of the band gap. (a) TM polarisation in rod-type PCs; (b) TE polarisation in hole-type PCs.

has much slower convergence with respect to the size of the supercell and the FEM mesh. In particular, the two modes which should be degenerate are actually noticeably different in Fig. 3 (b). For the FEM results presented in Fig. 3 (b) we use a supercell of size $9 \sqrt{3} \times 13$ and an FEM mesh with 52416 triangles and 26545 nodes (linear elements).

\section{Conclusion}

We have presented a major generalisation of the fictitious source superposition method so that it can deal with extended defects, rather than just simple point defects. This extension retains the key features of our earlier implementation and provides for the modelling of structures with a genuinely infinite cladding, making the use of a supercell unnecessary. Though here we used the fictitious sources to remove some inclusions completely or to change their refractive index, it is equally possible to change other properties of these inclusions more generally, for example their radii. We have also presented some of the first applications of the method and have demonstrated comparisons with other numerical techniques, exemplifying the efficiency and accuracy of extended FSS method.
This work was produced with the assistance of the Australian Research Council under the ARC Centres of Excellence program.

\section{References}

[1] J. D. Joannopoulos, R. D. Meade, J. N. Winn, Photonic Crystals: Molding the Flow of Light, Princeton University Press, 1995.

[2] S. Wilcox, L. C. Botten, R. C. McPhedran, C. G. Poulton, C. Martijn de Sterke, Phys. Rev. E 71 (2005) 056606.

[3] S. Wilcox, L. C. Botten, C. Martijn de Sterke, B. T. Kuhlmey, R. C. McPhedran, D. P. Fussell, S. Tomljenovic-Hanic, Opt. Express 13 (6) (2005) 1978.

[4] L. C. Botten, N.-A. P. Nicorovici, A. A. Asatryan, R. C. McPhedran, C. M. de Sterke, P. A. Robinson, J. Opt. Soc. Am. A 17 (12) (2000) 2165-2176.

[5] T. P. White, B. T. Kuhlmey, R. C. McPhedran, D. Maystre, G. Renversez, C. Martijn de Sterke, L. C. Botten, J. Opt. Soc. Am. B 19 (10) (2002) 2322-2330.

[6] L. C. Botten, N. A. Nicorovici, R. C. McPhedran, C. Martijn de Sterke, A. A. Asatryan, Phys. Rev. E 64 (2001) 046603

[7] L. C. Botten, K. B. Dossou, S. Wilcox, R. C. McPhedran, C. M. de Sterke, N. A. Nicorovici, A. A. Asatryan, Int. J. Microwave and Optical Technology 1 (1) (2006) 133-145.

[8] S. Wolfram, The Mathematica book, Wolfram Media / Cambridge University Press, 3rd edition, 1996.

[9] P. G. Ciarlet, The finite element method for elliptic problems, Society for Industrial and Applied Mathematics (SIAM), Philadelphia (USA), 2002.

[10] E. N. Economou, Green's Functions in Quantum Physics, p. 66, Springer-Verlag, Berlin, 1983. 